Revue d'histoire de l'Amérique française

REVUE D.HISTOIRE DE L'AMÉRIQUE FRANÇAISE

\title{
L'histoire de l'immigration au Québec : une invitation à fuir les ghettos
}

\section{Sylvie Taschereau}

Volume 41, numéro 4, printemps 1988

URI : https://id.erudit.org/iderudit/304617ar

DOI : https://doi.org/10.7202/304617ar

Aller au sommaire du numéro

Éditeur(s)

Institut d'histoire de l'Amérique française

ISSN

0035-2357 (imprimé)

1492-1383 (numérique)

Découvrir la revue

Citer cette note

Taschereau, S. (1988). L'histoire de l'immigration au Québec : une invitation à fuir les ghettos. Revue d'histoire de l'Amérique française, 41(4), 575-589.

https://doi.org/10.7202/304617ar d'utilisation que vous pouvez consulter en ligne.

https://apropos.erudit.org/fr/usagers/politique-dutilisation/ 
NOTE CRITIQUE

\title{
L'HISTOIRE DE L'IMMIGRATION AU QUÉBEC: UNE INVITATION À FUIR LES GHETTOS ${ }^{1}$
}

\author{
SYLVIE TASCHEREAU \\ Département d'histoire \\ Université du Québec à Montréal
}

Au cours des cinq ou dix dernières années, nous avons vu se multiplier au Québec les travaux portant sur l'immigration et sur les groupes ethniques. La production historiographique dans ce domaine n'est pas encore abondante, mais déjà son évolution ressemble fort à ce qu'elle a été d'abord aux États-Unis, puis au Canada anglais. Cela ne devrait pas nous étonner: cette nouvelle aire de spécialisation reflète des changements récents de notre société, changements qui, dans ce cas, se sont manifestés partout en Amérique du Nord.

Avant de nous intéresser à l'histoire de l'immigration telle qu'elle se fait au Québec, il convient d'évoquer ce contexte plus vaste dans lequel elle s'inscrit, puis de rappeler les particularités qu'on lui connaît ailleurs, voire les travers qu'on lui a reprochés jusqu'à présent. Le bilan qui suit est rapide: il s'agit moins de discuter les mérites et les faiblesses de chacun des travaux qui y sont mentionnés que d'en fournir une vue d'ensemble qui permette de suivre les directions qu'a prises cette recherche, de suggérer celles qu'elle pourrait prendre, enfin surtout de souligner l'apport qu'elle représente, tant pour l'ensemble de l'historiographie québécoise que pour celle de l'immigration. Un plaidoyer en faveur de l'histoire de l'immigration? Oui, sans doute. Et plus modestement, une interrogation: certes, les historiens québécois portent un intérêt croissant aux études dites «ethniques» et se montrent de plus en plus disposés à leur ménager une place - mais laquelle, au juste? Cette recherche ne doit ni ne peut évoluer en vase clos, et ses conclusions ne s'ajoutent pas simplement à ce que nous savons des populations qué-

\footnotetext{
1 Une première version de ce texte a été présentée à l'automne 1986, dans le cadre du congrès annuel de l'Institut d'histoire de l'Amérique française. Paul-André Linteau, Bruno Ramirez, Yves Otis et Johanne Collin ont bien voulu le lire et le commenter à différents moments de sa rédaction.
} 
bécoise ou canadienne, comme on ajoute une section à un recensement. Bien au contraire, les travaux parus à ce jour devraient nous en convaincre, sa contribution est active, elle peut apporter un éclairage nouveau non seulement aux dynamiques inter-ethniques, mais à tous les aspects de l'évolution de notre société.

Les années 1960-1970 ont été marquées en Amérique du Nord par un éveil de l'ethnicité dont le mouvement noir américain paraît avoir donné le signal de départ. Le phénomène prend des formes différentes aux États-Unis et au Canada, là plus militant, ici plus diffus; cependant il participe chaque fois à un même climat, à un moment où, un peu partout à travers le monde, se manifestent des mouvements ethniques, régionaux et nationaux de nature et d'importance diverses et où les rapports inter-ethniques semblent se modifier. En réalité, il serait peutêtre plus juste de parler d'une prise de conscience mutuelle, à la fois de la part de celles que l'on a appelé les minorités ethniques et de ceux qui se sont appelés les groupes fondateurs. Les premiers sont de plus en plus conscients du statut associé à leurs caractéristiques socio-culturelles, ils sont plus revendicateurs, surtout, en ce qui concerne leur identité et leurs droits d'autant que désormais leur poids démographique ne peut être ignoré. De fait, pour leur part, les groupes dominants d'origine française et britannique se sensibilisent davantage à ces réalités et à leurs implications pour l'avenir.

La reconnaissance de la pluralité ethnique et culturelle de la société canadienne est d'abord officialisée par la politique de multiculturalisme énoncée par le gouvernement fédéral en 1971 et suivie assez rapidement par les gouvernements du Manitoba, de l'Alberta et de l'Ontario. Au Québec, sans doute avons-nous été moins vite sensibilisés aux manifestations de ce changement: durant ces années, en effet, notre attention s'est plutôt portée vers le nationalisme franco-québécois. Mais il ne semble pas que l'expression de l'ethnicité chez les autres groupes ethniques s'en soit trouvée inhibée pour autant. Nous pourrions au contraire suggérer que le nationalisme franco-québécois des années 19601970 a été, dans cette province, l'un des catalyseurs de l'éveil de l'ethnicité. Ainsi, en 1968, les Italiens de Saint-Léonard expriment clairement leur refus de faire les frais des pressions à la francisation et de la bataille linguistique qu'elles entraînent. Plus récemment, la formation du mouvement «Alliance-Québec» est elle aussi, de toute évidence, le produit de ce contexte politique particulier.

Si le multiculturalisme peut être vu comme une réponse du Canada anglais au péril du séparatisme québécois, le gouvernement péquiste n'en a pas moins été amené à formuler lui-même un plan d'action à l'intention de celles que l'on a choisi d'appeler ici les communautés culturelles et qui n'est pas autre chose qu'une version québécoise de la 
politique fédérale ${ }^{2}$. De fait, l'immigration est plus que jamais reconnue comme un élément important de la stratégie de développement économique de nos pays. En même temps, le problème de la dénatalité qui préoccupe nos gouvernements depuis quelque temps déjà les porte à considérer tour à tour cette immigration comme une menace ou comme une solution: le problème est d'autant plus épineux au Québec qu'il s'ajoute à celui de l'anglicisation rapide des populations allophones. Enfin, comme cela s'est produit un peu partout en Amérique du Nord, l'évolution récente de la composition ethnique de la population québécoise est d'autant plus remarquée qu'elle est accompagnée de la montée d'un leadership ethnique plus articulé et plus revendicateur. Tous ces facteurs incitent le gouvernement québécois à s'assurer non seulement un contrôle sur l'immigration mais aussi - et il rejoint en cela les gouvernements des autres provinces - une certaine prise sur les relations inter-ethniques.

Tout naturellement, au moment où ils élaborent des politiques de plus en plus complexes pour gérer l'arrivée et l'intégration des populations nouvelles, de même que l'ensemble des rapports inter-ethniques, nos dirigeants se montrent extrêmement intéressés aux recherches menées dans ce domaine. Cet intérêt explique en partie le développement rapide des études ethniques aux États-Unis d'abord, puis au Canada anglais et au Québec.

Pendant toute la première moitié du 20e siècle, les travailleurs sociaux, économistes ou sociologues qui ont écrit sur le sujet ne se sont intéressés qu'aux aspects pathologiques de l'immigration. Le climat de l'après-guerre, et plus encore celui des années 1960-1970, favorise un changement d'attitude de la part des chercheurs, les amène à s'intéresser davantage au bagage socio-culturel des immigrants et aux particularités de l'établissement et du développement de chaque groupe. Pour leur part, les historiens nord-américains ne manifestent leur intérêt pour ces questions qu'après la Deuxième Guerre mondiale: la multiplication des travaux portant sur l'immigration est surtout le fait de la nouvelle histoire sociale, courant qui est souvent caractérisé par une approche multidisciplinaire.

Connaissant les antécédents de la recherche dans ce domaine, on comprend que cette historiographie soit tributaire des nombreuses études sociologiques qui l'ont précédée depuis les classiques de la sociologie américaine fonctionnaliste jusqu'aux analyses plus récentes influencées par les courants marxistes. En fait, elle continue à emprunter à cette discipline plusieurs de ses concepts, de même qu'elle s'inspire parfois de ses problématiques. Partout, plus encore là où elle débute, elle est

22 Sylvie Taschereau, «Un ministère en quête d'identité», entrevue avec Gérald Godin, ministre de l'Immigration et des Communautés culturelles, Quaderni culturali, 2 (1982). 
d'ailleurs assez souvent écrite par les sociologues eux-mêmes et, de plus en plus, par des chercheurs travaillant dans d'autres disciplines des sciences sociales.

Au Canada, cette tendance est particulièrement marquée dans le cas des études subventionnées par le gouvernement. Le meilleur exemple en est la série «Histoire des peuples du Canada/Generations», soutenue financièrement par le Secrétariat d'État pour donner suite aux recommandations de la Commission Laurendeau-Dunton: parmi les contributeurs de cette série d'histoire ethnique, les historiens ne sont pas plus nombreux que les sociologues et politicologues aux côtés desquels ils apparaissent. Sans doute cette particularité s'explique-t-elle d'abord par la jeunesse relative de ce champ d'étude en histoire. Par ailleurs, peut-être fait-on plus naturellement appel à des analystes de l'actualité pour étudier un phénomène dont les effets actuels et futurs inquiètent ou laissent perplexe. Mais le faible nombre des historiens de l'immigration trahit aussi, probablement, une certaine réticence de la part des chercheurs de cette discipline: il est vrai que l'on a souvent associé à l'histoire des communautés ethniques les tares d'une production qui est apparue sur ses traces ou qui l'a précédée et à propos de laquelle les anglophones parlent de filiopietism. Je fais référence au foisonnement d'écrits qui, sous des formes diverses, ont en commun, outre le goût de l'anecdote, le soin qu'ils mettent à glorifier le passé immigrant, à n'en retenir que ce qui confirme le mythe américain de réussite sociale.

Une des formes les plus courantes du filiopietism ${ }^{3}$ est l'inventaire que certains auteurs se croient obligés de faire de tous les cas de réussite sociale relevés parmi les membres de la communauté etnique qu'ils étudient; il se manifeste également dans l'insistance avec laquelle certains chercheurs font remonter l'histoire de cette communauté sur le continent nord-américain à l'époque des premiers explorateurs (les véritables découvreurs de l'Amérique se révèlent être alors, selon le cas, portugais, italiens, grecs ou même hongrois), ce qui a pour but de légitimer la présence et le statut de ladite communauté au sein de la société américaine, canadienne ou québécoise. Il faut souligner cependant que ces textes, issus des communautés ethniques elles-mêmes, sont une des expressions de ce phénomène difficile à saisir que l'on appelle ethnicité ${ }^{4}$ et, à ce titre, ils méritent certainement notre attention.

\footnotetext{
3 Roberto Perin en signale quelques cas parmi les ouvrages parus dans la série «Histoire des peuples du Canada", de même que dans celui de Antonio Alpalhao et Victor da Rosa, Les Portugais du Québec (Ottawa, 1979). Voir Roberto Perin, «Clio as an Ethnic: the Third Force in Canadian Historiography», Canadian Historical Review, 64,4 (1983): 441-467.

4 Après plusieurs années de débat, les experts ne sont pas encore parvenus à une définition unique et complète de ce que peut être l'ethnicité. Tel qu'il est utilisé dans ce texte, ce terme désigne l'appartenance ethnique, dans la mesure où celle-ci renvoie non seulement à un comportement social et culturel particulier, mais aussi à des caractéristiques socio-économiques et à un
} 
Le genre n'est d'ailleurs pas sans rappeler quelques exemples de notre petite histoire. De plus, si l'intérêt des gouvernements pour la recherche sur l'immigration a permis la réalisation de travaux très valables, il n'est pas tout à fait étranger à la perpétuation de ces écrits qu'encouragent des subventions à saveur électoraliste.

Les dangers de l'implication du gouvernement dans ce secteur de la recherche ont été dénoncés avec beaucoup de clairvoyance par Robert Harney $^{5}$. Parfois également, comme le déplore Roberto Perin ${ }^{6}$, l'intervention gouvernementale crée une pression à produire un texte à partir d'une recherche insuffisante avec, bien entendu, des résultats médiocres. Perin signale enfin un autre problème, plus sérieux encore et tout à fait indépendant cette fois de l'action des gouvernements: il s'agit de la tendance qu'ont certains auteurs à ignorer complètement le contexte historique dans lequel progresse la communauté qu'ils étudient. Le fait que ces auteurs ne soient pas des historiens ne peut excuser cette négligence ${ }^{7}$.

Ces tendances de même que ces travers se retrouvent dans l'historiographie québécoise sur l'immigration et sur les communautés ethniques. L'intérêt pour ces questions s'est affirmé ici un peu plus tard qu'en Ontario ou dans l'Ouest du pays. Peut-être, comme nous le suggérions plus tôt, notre attention a-t-elle été plus longtemps et plus exclusivement retenue par l'affrontement entre les deux «peuples fondateurs». Toutefois, si l'on tient compte du peu de rapports qu'entretiennent généralement les historiographies canadienne-anglaise et québécoise, ce décalage paraît moins remarquable, somme toute, que la similitude du développement de ce champ de la recherche historique chez les uns et les autres.

Tardif ou non, ce développement est bien amorcé. Encore une fois, l'implication des deux niveaux de gouvernement y a été très forte dès le départ: cela a eu pour effet de donner une impulsion importante à la recherche universitaire, permettant la réalisation de travaux dont les résultats sont stimulants, ou du moins prometteurs: ceux de Bruno Ramirez sur l'histoire sociale des Italiens de Montréal ${ }^{8}$, de même que ses recherches sur l'émigration des Canadiens français en Nouvelle-

statut qui résultent des dynamiques avec la société-hôte. Plus encore, comme cela a été suggéré dans les pages précédentes, il désigne ici la conscience plus ou moins aiguë de cette appartenance ethnique et de ses implications sociales, économiques et politiques.

5 Robert Harney, «Frozen Wastes: the State of Italian Canadian Studies», in S. Tomassi dir., Perspectives in Italian Immigration and Ethnicity (New York, 1977).

6 Roberto Perin, loc. cit., 445-446.

7 Ibid.

8 «Histoire sociale des Italiens de Montréal, 1870-1930», recherche subventionnée par le Secrétariat d'État. 
Angleterre 9 , ou encore l'étude de Denise Helly sur les Chinois de Montréal ${ }^{10}$, en sont de bons exemples. De même, au niveau provincial, si on ne retrouve pas au Québec l'équivalent de la Multicultural History Society of Ontario, par contre, à un niveau plus modeste, l'Institut québécois de recherche sur la culture a, jusqu'à présent, contribué à la publication de plusieurs livres et articles sur le sujet. Il semble bien cependant que l'on n'ait pas su éviter les effets négatifs de cette aide financière: le Québec a eu droit lui aussi à ses éléphants blancs de l'histoire de l'immigration. L'exemple le plus récent est une série d'ouvrages publiés chez Fides qui ressemble fort à un répertoire des communautés ethniques ayant pignon sur rue au Québec ${ }^{11}$. Cette série réunit à elle seule la plupart des problèmes évoqués plus tôt. Certes, on peut $\mathrm{y}$ trouver rapidement quelques indications sur l'importance démographique et la composition socio-économique de chacun des groupes dont on y traite. Mais la plupart des textes qui la composent restent superficiels, confondent trop souvent culture et folklore et présentent enfin une vision idyllique, ou du moins aseptisée des dynamiques inter-ethniques de la société québécoise.

Ce qui frappe davantage au Québec, plus encore que chez nos voisins du sud, c'est la pluridisciplinarité des études historiques, non seulement au niveau des concepts et des problématiques qu'elles utilisent, mais aussi en ce qui concerne leur écriture même. En fait, à ce stade-ci de la recherche, la contribution des sociologues et anthropologues paraît presque disproportionnée en regard d'une production d'ensemble qui est encore bien mince, il est vrai. Les ouvrages d'historiens sur l'immigration au Québec ne sont pas légion, on le sait: le petit livre de Bruno Ramirez sur les Italiens de Montréal ${ }^{12}$ est le premier qui soit issu du nouveau courant historiographique et le seul du genre. Par ailleurs, il s'agit moins du bilan d'une recherche achevée que d'une étude posant les bases d'une analyse historique du phénomène de l'immigration dans le cadre montréalais et québécois. Pour sa part, l'ouvrage de Ronald Rudin sur l'histoire du Québec anglophone ${ }^{13}$ n'est pas basé sur une recherche originale, bien que les précédentes recherches

\footnotetext{
9 «L'émigration des Canadiens français vers les États-Unis: analyse locale et comparée, 1870-1915», recherche subventionnée par le Conseil canadien de recherches en sciences humaines et sociales.

10 Recherche sur l'immigration chinoise au Québec, financée par Immigration-Canada (1982), le Secrétariat d'État (1983) et ACSAIR-FCAC (1984). Les résultats de cette recherche ont été publiés sous le titre Les Chinois de Montréal, 1877-1951 (Québec, Institut québécois de recherche sur la culture, 1987), grâce à l'aide financière de Multiculturalisme Canada.

11 «Les communautés culturelles du Québec», série dirigée par Michel Lefebvre et Yuri Orycshuck et subventionnée par le Secrétariat d'État. Montréal, Fides, 1986-1987.

${ }_{12}$ Bruno Ramirez, Les premiers Italiens de Montréal: l'origine de la Petite Italie du Québec (Montréal, Boréal Express, 1984).

${ }_{13}$ Ronald Rudin, Histoire du Québec anglophone, 1759-1980 (Québec, Institut québécois de recherche sur la culture, 1986).
} 
de cet auteur y soient mises à contribution. Cependant, cette synthèse est bienvenue dans un domaine où, même à ce stade initial de la recherche, il est très utile de faire le point sur ce que nous savons.

S'agissant des anglophones du Québec et des relations entre Canadiens anglais et Canadiens français, nous pourrions nous croire en terrain connu. Mais Rudin nous oblige à corriger notre vision de «la» communauté anglophone, que l'historiographie absorbée, sans doute, par la dynamique existant entre celle-ci et les francophones présente trop souvent comme un groupe homogène. Rappelant ce que quelques auteurs ont signalé de façon ponctuelle ${ }^{14}$, il fait apparaître non pas une mais plusieurs communautés que distingue l'appartenance ethnique, religieuse ou de classe, ces divisions pouvant se recouper et évoluer entre le $18 \mathrm{e}$ et le $20 \mathrm{e}$ siècle. De fait, ajoute Rudin, ces communautés, en proie à des tensions multiples, présentent rarement un front commun contre les francophones et se découvrent à l'occasion certaines affinités avec ces derniers. Des travaux futurs viendront, espérons-le, étayer, contredire ou nuancer l'une ou l'autre des conclusions avancées dans ce livre. Il reste que cette mise au point quant à la diversité du groupe anglophone prépare le terrain à l'étude de l'ensemble des relations interethniques au Québec dont les dynamiques entre anglophones et francophones forment en partie la toile de fond.

Exception faite de l'ouvrage de Rudin et de celui de Ramirez, les travaux d'historiens portant sur l'immigration et les communautés ethniques n'ont été publiés jusqu'à présent que sous forme d'articles et de notes de recherche parus, pour la plupart, dans des revues canadiennes. Encore une fois, sur la scène québécoise, la présence des historiens se fait moins sentir que celle des chercheurs des autres disciplines.

Les résultats de cette écriture pluridisciplinaire de l'histoire de l'immigration ne sont pas également convaincants. Mais en somme, il appartient aux historiens de profiter de l'excellent terrain d'échanges qu'offre ce champ de la recherche et de s'inspirer de ces travaux ou, au contraire, de corriger le tir. Rudin et Ramirez ne sont d'ailleurs pas les seuls, parmi ces derniers, à s'être montrés attentifs aux études ethniques. Paul-André Linteau est l'un des premiers à s'y être intéressé: il l'a fait d'abord en tant que spécialiste d'histoire urbaine ${ }^{15}$; il a également contribué à son progrès en y orientant et en encourageant les efforts de plusieurs jeunes chercheurs et chercheures. Gerald Tulchinsky, historien économiste bien connu, s'y est impliqué plus direc-

14 Voir notamment l'article de Robert Sweeny, «Esquisse de l'histoire économique du Québec anglophone», dans Gary Caldwell et Eric Waddell, dir., Les anglophones du Québec: de majoritaires à minoritaires (Québec, Institut québécois de recherche sur la culture, 1982), 73-92. 15 Paul-André Linteau, «La montée du cosmopolitanisme montréalais», Questions de culture, 2 (1982): 23-54. 
tement: depuis quelques années, en effet, il étudie la communauté juive de Montréal ${ }^{16}$. Il faut enfin souligner le fait que la plus récente synthèse d'histoire économique et sociale du Québec consacre une ou deux sections, dans chacun de ses deux volumes, à l'immigration et aux communautés ethniques, ce qui confirme sans aucun doute l'intérêt porté à ces questions ${ }^{17}$. Dans ce cas, ce sont surtout les aspects démographique et linguistique de ces phénomènes, jusqu'ici plus étudiés, qui retiennent l'attention des auteurs. Ces derniers nous rappellent toutefois brièvement, dans l'un et l'autre volumes, les caractéristiques socio-économiques des communautés les plus importantes. En effet, nous disentils, il ne saurait être question, dans un ouvrage du genre, de présenter l'histoire de chacun de ces groupes ${ }^{18}$. Nous pourrions ajouter pour notre part que ce ne serait d'ailleurs pas la meilleure façon d'aborder cette matière.

Sans doute l'état actuel de nos connaissances ne nous permet-il pas d'intégrer les résultats de ces recherches à chacun des chapitres d'une histoire-synthèse. Pourtant, les phénomènes migratoires et les dynamiques inter-ethniques qui se manifestent au Québec ne constituent pas une section «à part» de notre histoire. Nous devons souhaiter que les recherches futures nous amènent à rendre compte de la diversité de leurs effets et de ce que ces dynamiques nous révèlent de notre société. Il est un peu tôt pour tracer un véritable bilan de l'historiographie de l'immigration au Québec. Cependant, après avoir rappelé les directions qu'elle a prises jusqu'à présent, le moment est peut-être bien choisi pour réfléchir aux voies qui s'offrent à elle, à la place qu'elle devrait occuper dans l'histoire du Québec et à sa contribution à l'histoire nord-américaine de l'immigration et des communautés ethniques.

Pas plus que les autres disciplines des sciences sociales l'histoire n'échappe au problème du cloisonnement qui tend à s'établir entre ses différentes aires de spécialisation. Or, la définition d'un nouveau champ d'étude comporte chaque fois le risque de fragmenter davantage l'une ou l'autre de ces aires. Ce n'est évidemment pas la pertinence de la spécialisation qui est ici en cause, mais plutôt notre habileté à approfondir ces recherches tout en gardant une vision d'ensemble de leurs implications et en leur assurant la place qui leur revient dans notre historiographie. En ce qui les concerne, les historiens de l'immigration

\footnotetext{
16 Gerald Tulchinsky, «Immigration and Charity in the Montreal Jewish Community before 1890», Histoire sociale/Social History, 16,32 (1983): 359-380; «The Third Solitude: A. M. Klein's Jewish Montreal, 1910-1950», Journal of Canadian Studies, 19,2 (1984): 96-112.

17 Paul-André Linteau, René Durocher et Jean-Claude Robert, Histoire du Québec contemporain, 1: De la Confédération à la Crise (Montréal, Boréal Express, 1979), chapitres 2 et 3; Paul-André Linteau, René Durocher et Jean-Claude Robert et François Ricard, 2: Le Québec depuis 1930 (Montréal, Boréal, 1986), chapitres 15, 40 et 41.

18 Ibid., 2: 535.
} 
ont d'autant plus de raisons de craindre les espaces clos - les ghettos, autrement dit - que plusieurs ouvrages portant sur l'histoire d'une communauté ethnique s'y sont déjà embourbés, cédant à l'excès du filiopietism ou menant avec zèle un récit ahistorique.

De fait, en partie pour ces motifs, ces recherches suggèrent encore d'emblée à beaucoup d'historiens une image de superficialité, je serais tentée de dire de folklore. Cela explique que plusieurs de ceux qui s'y intéressent éprouvent parfois le besoin de légitimer son existence. L'exercice en soi n'est pas inutile et s'inscrit probablement dans le processus normal de définition d'un champ d'étude. C'est sans doute ce qui amène Roberto Perin à insister sur la distinction qui doit être faite entre l'histoire de l'immigration et des communautés immigrantes ${ }^{19}$ et celle de l'ensemble de la population canadienne: nier cette distinction, dit-il en substance ${ }^{20}$, c'est, ironiquement, reprendre à son compte l'argument des détracteurs de l'histoire de l'immigration. Peut-être. Pour ma part, je ne m'inquièterais pas de faire valoir à quel point cette histoire est partie intégrante de celles des sociétés canadienne et québécoise: à mon sens, c'est précisément pour cela qu'elle ne peut être ignorée.

En effet, l'étude sérieuse et complète d'un groupe immigrant ou d'une communauté ethnique en Amérique du Nord exige à la fois que l'on connaisse ses origines et le contexte socio-économique et historique particulier dans lequel ce groupe ou cette communauté évolue. Par ailleurs, quel que soit l'intérêt que présente l'étude d'un groupe ou d'une communauté donnés, une telle recherche est d'autant plus valable qu'elle nous apprend quelque chose, ou nous amène à nous interroger sur l'ensemble de la société à laquelle les immigrants s'intègrent et participent en tant qu'acteurs sociaux. Les résultats de plusieurs des travaux entrepris au Québec au cours des dernières années ne peuvent qu'affermir cette conviction.

19 R. Perin éprouve une certaine réticence à parler de groupes ou de communautés ethniques dans le contexte nord-américain (loc.cit., 443-444) et préfère utiliser l'expression «communautés immigrantes». Cependant, si cette expression évoque les débuts de l'établissement des immigrants et de la communauté qu'ils créent (la population immigrante non encore établie peut être vue comme formant un ou des groupes immigrants), elle me paraît moins apte à définir des communautés plus anciennes. Il est vrai que les recherches historiques concernent souvent des groupes immigrants et des communautés immigrantes. Toutefois, l'étude d'une communauté en formation, pour peu qu'elle s'étende sur quelques années, porte nécessairement sur un nombre croissant d'individus nés dans le pays-hôte. La distinction entre les uns et les autres n'est pas toujours clairement établie par les chercheurs; par contre, certaines observations, telle la constitution des familles ou la mobilité inter-générationnelle, la rendent indispensable. En ce qui concerne la réalité nord-américaine des «groupes ethniques» de même que celle de ce que l'on a appelé l'ethnicité, voir notamment l'article de Stefanos Constantinides, «De l'autonomie relative de l'ethnicité en tant que construit idéologique», Études ethniques au Canada, 18,2 (1986): 102-114.

Roberto Perin, loc. cit., 444. 
Les contributions de ces travaux sont nombreuses. Bien que la plupart portent sur des populations venus de l'étranger, il est important de rappeler que notre connaissance des phénomènes migratoires régionaux et nationaux profite directement de ces recherches: c'est dire qu'elles concernent doublement la population québécoise. En effet, si dans la première moitié de ce siècle et jusqu'aux récentes décennies, l'impact de l'immigration a été moins senti au Québec qu'il ne l'a été dans l'ouest du pays, par exemple, est-il besoin de rappeler que cette province est, entre toutes, celle qui a connu la plus forte émigration de Canadiens vers les États-Unis? La progression de l'histoire de l'immigration a permis de poser un regard neuf sur ce phénomène dont l'ampleur a été mesurée, certes, mais dont les causes sont, jusqu'à récemment, demeurées assez nébuleuses. Les travaux menés aux Etats-Unis ${ }^{21}$, puis au Québec ${ }^{22}$, nous ont fait mieux connaître la nature, l'étendue et le fonctionnement des réseaux migratoires des deux côtés de la frontière.

Plusieurs des recherches récentes concernent les aspects économiques de l'immigration et du développement des communautés immigrantes; ce faisant, elles nous amènent nécessairement à observer les rouages de notre économie ou même ses interstices. C'est Robert Harney qui le premier a étudié le «commerce de migration» qu'engendrent le transport et l'arrivée massive de travailleurs immigrants à Montréal au début du siècle ${ }^{23}$. Bruno Ramirez a pour sa part retracé le réseau d'échange de services créé par l'établissement des Italiens dans cette ville: il nous a révélé ainsi l'existence d'une forme d'économie parallèle, s'appuyant sur une éthique de comportement que partagent les immigrants de même origine ${ }^{24}$. Cet historien a également cherché à mieux comprendre l'évolution simultanée et en apparence paradoxale, d'un fort courant d'émigration québécoise vers la Nouvelle-Angleterre et d'une immigration étrangère dirigée essentiellement vers Montréal ${ }^{25}$.

21 Voir en particulier l'ouvrage de Tamara K. Hareven, Family Time and Industrial Time: the Relationship Between Family and Work in a New England Industrial Community (Cambridge, Mass., Harvard University Press, 1982).

22 Jacques Rouillard, Ah! Les États! (Montréal, Boréal Express, 1985); Bruno Ramirez, «A Socio-Economic Profile of the French-Canadian Labour Force in the New England Cotton Industry during the Progressive Era», Labour/Le travailleur, 11 (Spring 1983): 125-142; Bruno Ramirez et Jean Lamarre, «Du Québec vers les États-Unis: l'étude des lieux d'origine», Revue d' histoire de l'Amérique française, 38,3 (hiver 1985): 409-422.

${ }_{23}$ Robert F. Harney, "The Padrone and the Immigrant», Canadian Review of American Studies, 5 (1974): 101-118. Voir également «Montreal's King of Immigrant Labour: a Case Study on Padronism», Labour/Le travailleur, 4 (1979): 57-84.

24 Bruno Ramirez, «The Socio-Economy of Settlement: Some Historical Hypotheses», Revue d'histoire urbaine/Urban History Review (1982).

25 Bruno Ramirez, «The Crossroad Province: Quebec's Place in International Migrations, 1870-1915», communication présentée dans le cadre de la conférence internationale «A Century of European Migrations, 1830-1930, in Comparative Perspective», qui a eu lieu à Saint Paul, Minnesota, novembre 1986. 
S'appuyant à la fois sur ses connaissances du mouvement d'émigration des Canadiens français et sur son observation des travailleurs migrants et immigrants italiens venus au Québec, il élabore une problématique centrée sur l'articulation entre mouvements migratoires et marché du travail national ou régional et met en relief l'importance du phénomène de segmentation de ces marchés suivant des lignes ethniques. C'est ce même phénomène, ainsi que les dynamiques qui le sous-tendent, que met en lumière la recherche de Denise Helly sur les Chinois vivant dans la métropole québécoise entre 1877 et $1951^{26}$.

La lecture de ces travaux suggère une ébauche de comparaison: comme cela paraît être le cas pour les Canadiens français et pour les Italiens, les caractéristiques socio-économiques et le mode d'immigration des Chinois orientent le choix de leurs activités dans la ville. Cette fois cependant, le rôle que la société-hôte réserve aux travailleurs immigrants et les pressions qu'elle exerce sur eux sont plus évidents et ils imposent de façon plus brutale les limites de leur participation à la vie montréalaise. L'ensemble de ces facteurs explique qu'à la fin du siècle dernier et dans toute la première moitié du $20 \mathrm{e}$ siècle, la majorité des Chinois de Montréal ait été confinée au commerce de la buanderie.

C'est également le préjugé ethnique et racial qui encourage la création d'une catégorie d'emplois «réservés» aux Noirs engagés par le CPR et le CNR: plusieurs de ces travailleurs s'établissent à Montréal où ils représentent, jusqu'aux années 1950, une forte proportion de la communauté noire. Encore une fois, ces préjugés sont d'autant plus virulents qu'ils desservent des intérêts économiques: ceux de l'employeur, avant tout, mais aussi ceux des employés blancs des compagnies ferroviaires, qui croient avoir avantage à maintenir ces ghettos ${ }^{27}$.

En somme, suivre l'itinéraire des travailleurs immigrants dans cette ville, comme celui des Canadiens français en Nouvelle-Angleterre, nous amène à poser un regard avec un éclairage particulier, soit, mais sous plusieurs angles, sur un phénomène dont, en ce qui concerne la première moitié de ce siècle, nous connaissons encore bien peu de choses: les transformations du marché du travail.

Une immigration importante peut influencer la stratification et l'organisation de ce marché; d'ailleurs elle devient souvent, au Canada comme aux États-Unis, un élément important de la stratégie patronale; enfin ses effets sur le comportement ouvrier sont multiples et parfois contradictoires. Aussi serait-il bon que nous nous interrogions sur les dynamiques inter-ethniques qui se manifestent au sein des organisations

26 Denise Helly, Les Chinois de Montréal, 1877-1951 (Québec, Institut québécois de recherche sur la culture, 1987).

27 Agnes Calliste, «Sleeping Car Porters in Canada: an Ethnically Submerged Split Labour Market», Etudes ethniques au Canada, 19,1 (1987): 2-20. 
ouvrières et dans l'ensemble du mouvement ouvrier. Mis à part l'article d'Agnes Calliste mentionné ci-dessus, les rares travaux qui ont été faits dans ce sens portent sur les immigrants juifs arrivés entre la fin du $19 \mathrm{e}$ siècle et le début du $20 \mathrm{e}$ siècle et dont beaucoup ont travaillé dans l'industrie du vêtement ${ }^{28}$. C'est peu, surtout lorsqu'on songe que la plupart des immigrants sont venus gonfler les rangs de la classe ouvrière.

De la même façon, si nous découvrons ou redécouvrons l'importance du vote ethnique au Québec, la venue d'immigrants et l'existence de communautés ethniques ont vraisemblablement influencé de beaucoup d'autres manières la vie politique québécoise. Par exemple, à travers la création et l'évolution de partis politiques: non pas tant ceux des grands partis traditionnels (ni même, à la différence de ce qui se passe dans l'Ouest, du CCF), mais plutôt ceux de formations plus modestes ou marginales, telles le Parti communiste, les partis et mouvements fascistes ou nazis. Roberto Perin s'est avancé sur ce terrain en étudiant l'émergence d'un mouvement fasciste dans la communauté italienne de Montréal et le double jeu des représentants du gouvernement italien auprès des autorités canadiennes et québécoises ${ }^{29}$. De façon plus diffuse, directement ou indirectement, les expériences de lutte politique et ouvrière qui font partie du bagage de certains immigrants ont pu marquer elles aussi la scène politique de cette province. Les Irlandais nous fournissent à eux seuls plusieurs exemples de cette continuité: le cas des Féniens au Canada, et en partie au Québec, a été étudié par l'historien Hereward Senior ${ }^{30}$; celui des Orangistes est surtout connu en Ontario et dans les Maritimes ${ }^{31}$. Mais il est aussi permis d'évoquer les trade-unionistes anglais et les bundistes ou les travaillistes sionistes juifs dont les activités ont été suivies ailleurs au Canada ou aux ÉtatsUnis $^{32}$. Beaucoup d'autres avenues restent encore à explorer: quel a été, par exemple, l'impact de l'immigration sur l'évolution de l'éspace urbain $^{33}$ ? Nous connaissons partiellement l'oeuvre sociale des Églises

28 Voir l'ouvrage édité par Pierre Anctil et Gary Caldwell, Juifs et réalités juives au Québec (Québec, Institut de recherche sur la culture, 1983), en particulier la contribution de Alexandra Szacka, qui est également l'auteure d'un mémoire non publié en anthropologie, Ethnicité et frag. mentation du mouvement ouvrier: les immigrants juifs au Québec, 1920-1940 (Université Laval, 1981).

29 Roberto Perin, «Conflits d'identité et d'allégeance: la propagande du consulat italien à Montréal dans les années 1930», Questions de culture, 2 (1982): 81-102.

30 Hereward Senior, The Fenians in Canada (Toronto, MacMillan, 1978).

31 Voir notamment Gregory S. Kealey, «The Orange Order in Toronto», in Essays in Working Class History (Toronto, McClelland and Stewart, 1976); Scott W. See, "The Orange and Social Violence in Mid-Nineteenth Century Saint John», Acadiensis, 13,1 (automne 1983): 6892.

32 Elles sont évoquées dans l'ouvrage de Pierre Anctil et Gary Caldwell, op.cit. Voir également A. Ross McCormack, «British Working-Class Immigrants and Canadian Radicalism: the Case of Arthur Puttee», Études ethniques au Canada, 19,2 (1982): 23-37.

33 Les choix et les patterns résidentiels des communautés ethniques constituent un aspect de cette question: à ce propos, l'impressionnant travail complété récemment par Claire McNicoll représente une contribution importante de même qu'une base précieuse aux études des dynamiques 
protestantes auprès de ces immigrants, mais qu'en est-il de l'Église catholique $^{34}$ ? De même, les études amorcées sur les multiples institutions juives qui apparaissent à Montréal dès la fin du $19 \mathrm{e}$ siècle ${ }^{35}$ doiventelles être suivies d'autres travaux qui nous permettront de mieux apprécier le terrain social et institutionnel créé par l'immigration et, à la suite de Barbara Roberts ${ }^{36}$, de mieux connaître les enjeux des conflits et des luttes éventuels qui y éclatent.

Outre le risque de ghettoïsation évoqué plus tôt, d'autres cloisonnements, bien sûr, pourraient entraver l'essor de l'histoire de l'immigration au Québec. Son héritage particulier et la part que prennent à son écriture anthropologues et sociologues lui assurent en principe une ouverture aux autres sciences sociales. Nous pourrions nous demander si les emprunts faits à ces disciplines et ceux que lui font parfois ces dernières tiennent vraiment du dialogue: les termes et les limites de cette interaction méritent en effet d'être étudiés de façon particulière, mais ce n'est pas ici notre propos.

Le parallèle tracé plus haut entre le développement de l'historiographie québécoise de l'immigration et celui qui l'a précédé aux ÉtatsUnis et au Canada laisse deviner la très grande influence que ce dernier pourrait avoir sur les problématiques et la méthodologie adoptées par les chercheurs québécois. Comme toute approche empruntée à une historiographie étrangère ou autre, cela ne risque-t-il pas de mener à l'application de modèles inadéquats?

En réalité, il est un peu tôt pour mesurer l'empreinte de l'historiographie américaine, comme il est trop tôt pour juger de celle de la sociologie et de l'anthropologie québécoises, elles-mêmes traversées par des courants divers. Un fait demeure: impossible de poursuivre ici des études sur l'immigration sans tenir compte de l'impressionnante production qui existe chez nos voisins. Mais pour peu que nous soyons conscients de la spécificité québécoise, le poids de cette littérature ne devrait pas gêner la conception de problématiques originales. Par ailleurs, si ce domaine de notre historiographie risque d'être celui où l'influence nord-américaine sera la plus sentie, il est aussi probablement celui qui se prête le plus naturellement à l'analyse comparative. Sans doute, avant de s'engager dans cette voie, convient-il de multiplier les

inter-ethniques dans la ville. Claire McNicoll, L'évolution spatiale des groupes ethniques à Montréal, 1871-1981, thèse de doctorat en géographie, École des hautes études en sciences sociales, Paris, 1986.

34 L'ouvrage de Denise Helly présenté plus haut offre un début de réponse à cette question. Voir également Fernand Harvey, «L'ouverture du Québec au multiculturalisme, (1900-1981)», Revue française d'études canadiennes, 21,2 (1986): 219-228.

35 Pierre Anctil et Gary Caldwell, op. cit.; Gerald Tulchinsky, op. cit.

36 Barbara Roberts, «Sex, Politics, and Religion: Controversies in Female Reform Work in Montreal, 1881-1919», Atlantis, 6,2 (automne 1981): 25-38. 
études locales et régionales. Mais ceci dit, l'observation du phénomène migratoire s'accommode mal du découpage des frontières provinciales ou même nationales: les itinéraires immigrants sur ce continent et les mouvements constants de populations de part et d'autre de la frontière canado-américaine nous le prouvent. En fait, l'élaboration d'une approche comparative de l'impact de l'immigration, de l'évolution des communautés ethniques, de l'accueil fait à ces populations nouvelles et de leurs rapports avec la société-hôte devraient aider non pas tant à la réalisation d'une histoire canadienne ou nord-américaine de l'immigration, mais bien davantage à saisir les spécificités des sociétés québécoise, canadienne ou américaine. Quels que soient les débats qu'elles soulèvent, et d'ailleurs en partie parce qu'elles soulèvent des débats, des études comme celles que Donald Akenson a menées sur les Irlandais en Ontario ${ }^{37}$, soulignant ce qui les différencie, en termes de caractéristiques socio-économiques, de leurs compatriotes établis aux ÉtatsUnis, enrichissent non seulement notre compréhension de ce mouvement en Amérique du Nord mais peuvent de surcroît suggérer des pistes intéressantes, notamment en ce qui a trait au développement économique des pays ou régions concernés. Dans quelle mesure, par exemple, les différences qu'observe cet auteur reflètent-t-elles les particularités des marchés du travail régionaux et nationaux?

Toute réflexion sur l'immigration au Québec nous ramène tôt ou tard à la question nationale ${ }^{38}$. Plus généralement, la très vaste question des rapports de pouvoir entre majorité et minorité se pose dans des termes sensiblement différents selon qu'on se situe aux États-Unis ou au Canada, au Québec ou dans une autre province. Vu sous cette angle, l'accueil réservé aux immigrants reçoit un éclairage particulier et fort intéressant. Historiquement, la perception que différents groupes de la société québécoise peuvent avoir de leur statut dans l'ensemble de la société canadienne influence certainement leur attitude face à l'immigration. Mais une analyse systématique du ou des discours sur l'immigration, de même que des attitudes des Québécois face au phénomène migratoire et aux communautés ethniques qui se forment ici, devrait également les situer dans le contexte plus large du Canada et de l'Amérique du Nord: en quoi ces discours diffèrent-ils de ceux qui s'élaborent en Ontario, dans les Maritimes ou dans les provinces de

\footnotetext{
37 Donald H. Akenson, The Irish in Ontario: A Study in Rural History, Montréal/Kingston, McGill/Queen's University Press, 1984.

${ }_{38}$ Ainsi, les sociologues et politologues québécois ont-ils axé cette réflexion en particulier autour du débat linguistique. A ce propos, voir par exemple Harold M. Waller et Morton Weinfeld, «The Jews of Quebec and le fait français», in The Canadian Jewish Mosaic, Toronto, 1981:415439, de même que l'ouvrage de Pierre Anctil et Gary Caldwell mentionné plus tôt, Juifs et réalités juives...; voir également:Sociologie et sociétés, XV, 2, (1983); ou encore, en ce qui concerne les Italiens, l'étude récemment parue de Donat Taddeo et Raymond Taras, Le débat linguistique au Québec, (Montréal, P.U.M., 1987), qui adopte en partie une approche historique.
} 
l'Ouest? Quelles formes particulières le nativisme, et plus généralement l'attitude et le sentiment anti-immigrant, prennent-ils ici et pourquoi?

Le fait d'établir ce type de dialogue nous éviterait enfin de voir trop souvent des historiens canadiens contourner soigneusement le territoire québécois en abordant la question de l'immigration au Canada, comme le fait Donald Avery lorsqu'il écrit son intéressant petit ouvrage «Dangerous Foreigners»" ${ }^{39}$. Il est vrai que cette démarche est on ne peut plus symptomatique de l'ensemble de l'historiographie canadienne. Qui sait? Voilà peut-être une bonne occasion de rompre avec de vieilles habitudes et de créer un lien entre des historiographies dont les échanges, rares et sélectifs, offrent de part et d'autre une vision tronquée, souvent vieillie, de la recherche.

39 Donald Avery, «Dangerous Foreigners»: European Immigrant Workers and Labour Radicalism in Canada, 1896-1932 (Toronto, McClelland and Stewart, 1979). 